\title{
Characterization of a novel model incorporating airway epithelial damage and related fibrosis to the pathogenesis of asthma
}

\author{
Simon G Royce ${ }^{1}$, Krupesh P Patel ${ }^{1}$ and Chrishan S Samuel
}

Asthma develops from injury to the airways/lungs, stemming from airway inflammation (Al) and airway remodeling (AWR), both contributing to airway hyperresponsiveness (AHR). Airway epithelial damage has been identified as a new etiology of asthma but is not targeted by current treatments. Furthermore, it is poorly studied in currently used animal models of AI and AWR. Therefore, this study aimed to incorporate epithelial damage/repair with the well-established ovalbumin (OVA)-induced model of chronic allergic airway disease (AAD), which presents with Al, AWR, and AHR, mimicking several features of human asthma. A 3-day naphthalene (NA)-induced model of epithelial damage/repair was superimposed onto the 9-week OVA-induced model of chronic AAD, before 6 weeks of OVA nebulization (NA + OVA group), during the second last OVA nebulization period (OVA/NA group) or 1 day after the 6-week OVA nebulization period (OVA + NA group), using 6-8-week-old female Balb/c mice ( $n=6-12 /$ group). Mice subjected to the 9-week OVA model, 3-day NA model or respective vehicle treatments (saline and corn oil) were used as appropriate controls. OVA alone significantly increased epithelial thickness and apoptosis, goblet cell metaplasia, TGF- $\beta 1$, subepithelial collagen (assessed by morphometric analyses of various histological stains), total lung collagen (hydroxyproline analysis), and AHR (invasive plethysmography) compared with that in saline-treated mice (all $P<0.05$ vs saline treatment). NA alone caused a significant increase in epithelial denudation and apoptosis, TGF- $\beta 1$, subepithelial, and total lung collagen compared with respective measurements from corn oil-treated controls (all $P<0.01$ vs corn oil treatment). All three combined models underwent varying degrees of epithelial damage and AWR, with the OVA + NA model demonstrating the greatest increase in subepithelial/total lung collagen and AHR (all $P<0.05$ vs OVA alone or NA alone). These combined models of airway epithelial damage/AAD demonstrated that epithelial damage is a key contributor to AWR, fibrosis and related AHR, and augments the effects of $\mathrm{Al}$ on these parameters.

Laboratory Investigation (2014) 94, 1326-1339; doi:10.1038/labinvest.2014.119; published online 29 September 2014

Asthma is a chronic inflammatory airway disease. According to the World Health Organization, 300 million people worldwide suffer from this disease, equating to $1 / 250$ deaths, and it is the most prevalent chronic disease affecting children. ${ }^{1}$ There are three central components involved in the pathogenesis of asthma: airway inflammation (AI), airway remodeling (AWR), and airway hyperresponsiveness (AHR). ${ }^{1}$ Repeated episodes of AI lead to AHR and may initiate irreversible structural changes in the airways (AWR). ${ }^{1}$ However, studies have shown that AWR can also occur early in asthma pathogenesis and significantly contribute to AHR independently of AI. ${ }^{2}$ Epithelial damage is a process that is emerging as a key cause of AWR, and has been shown to expose the epithelial nerves to specific or nonspecific stimuli in patients with mild and severe AHR. ${ }^{3,4}$ The epithelium is also an important physical barrier protecting the airway from pathogenic organisms and harmful substances that may be inhaled, while also having an important role in innate and adaptive immune function. ${ }^{3}$ Genetic mutations can lead to the susceptibility of epithelial damage. ${ }^{5}$ For example, a mutation or disruption to the E-cadherin gene, $\mathrm{CDH} 1$, leads to a reduction in E-cadherin levels. ${ }^{6}$ This may be followed by denudation (shedding) of the epithelium, which leads to re-epithelialization, alterations in the cell types that make up the epithelium and aberrant wound healing. ${ }^{7,8}$ 
Current asthma therapies work by either supressing $\mathrm{AHR}^{9}$ or inhibiting AI. ${ }^{10}$ There are, however, several shortcomings of these currently used treatments for asthma. In the case of short- and long-acting $\beta$-agonists, even though they relieve AHR transiently, they do not supress the processes that are involved in AI and AWR, which are the two main contributors to AHR. ${ }^{9}$ Likewise, while corticosteroids inhibit $\mathrm{AI}$ and the contributions of AI to AHR, they only mildly affect AWR and, hence, AWR-induced AHR. Thus, to better develop novel treatments that can target all three central features of asthma, more research must be done in order to better understand the pathogenesis of asthma.

The limited availability of tissue and primary cells from the human airways has led many investigators to explore the potential of experimental models that can mimic the processes of human asthma. The experimental form of asthma that is induced in laboratory animals is allergic airways disease (AAD). Although all models of AAD exhibit several features of human asthma, none of these undergo the full spectrum of features seen in human disease. Currently there are several experimental models of ovalbumin (OVA)induced AAD that have different time frames. ${ }^{11}$ Acute and subacute models of AAD, which can be established over 3-4 and 7 days, respectively, undergo AI and some AWR, but do not present with airway fibrosis, which is a well-established hallmark of asthma. ${ }^{11,12}$ On the other hand, chronic models of AAD exhibit AI, several features of AWR (epithelial thickening, goblet cell metaplasia) including fibrosis, an increase in the expression of pro-fibrotic factors and AHR, ${ }^{11}$ but do not undergo severe epithelial damage. ${ }^{11}$ There are currently several acute experimental animal models of epithelial damage, each using a different toxin to cause epithelial damage. ${ }^{13}$ The best characterized is naphthalene (NA), a Clara cell-specific cytotoxicant. ${ }^{14}$ Clara cells are located on the bronchial epithelium ${ }^{15,16}$ and are responsible for the secretion of various products into the bronchial lining that are important for the protection of the epithelium (such as surfactant proteins) ${ }^{15,17}$ as well as inhibiting inflammatory reactions mediated via the Th2 response. ${ }^{15,17}$

Our hypothesis was that superimposing the NA-induced model of epithelial damage onto the gold standard chronic OVA-induced model of AAD would exhibit a wider spectrum of features that were associated with asthma (ie, AI, airway epithelial damage, airway fibrosis, and AHR) and that may be used in the future as a better representative experimental model of human asthma. Therefore this study aimed to superimpose the NA-induced mouse model of epithelial damage onto the 9-week OVA-induced mouse model of AAD at three distinct time points to (1) determine how closely these combined models represented the features of human asthma and (2) use these models to determine the contributions of AI vs epithelial damage to fibrosis and AHR, which are the hallmarks of asthma.

\section{MATERIALS AND METHODS \\ Animals}

Six- to eight-week-old female Balb/c wild-type mice (provided by Monash University Animal Services) were allowed to acclimatize for at least 4 days prior to any experimentation and maintained on a 12-h light:dark cycle with free access to standard rodent chow (Barastoc Stockfeeds, Pakenham, VIC, Australia) and water. Female Balb/c wild-type mice have been shown to be more prone to a Th2 response and undergo higher airway reactivity (in response to allergens) compared with their male counterparts and other commonly used murine strains, ${ }^{18,19}$ and as such have been used extensively in chronic AAD models. ${ }^{20,21}$ All experimental procedures were performed according to the regulations approved by the Monash University Animal Ethics Committee, which adheres to the Australian Guidelines for the Care and Use of Laboratory Animal for Scientific Purposes.

\section{Establishing the Combined Models of AAD}

Mice were subjected to three combined models of AAD: all mice were exposed to the 9-week OVA-induced model of chronic AI-induced AAD, as described previously. ${ }^{22}$ Briefly, mice $(n=32)$ were sensitized with two i.p. injections of $10 \mu \mathrm{g}$ Grade V OVA (Sigma-Aldrich, St Louis, MO, USA) and $1 \mathrm{mg}$ aluminum potassium sulfate adjuvant (alum; AJAX Chemicals, Kotara, NSW, Australia) in $0.5 \mathrm{ml}$ of saline on day 0 and day 14. They were then subjected to nebulization (inhalation of an aerosol) with $2.5 \%$ (w/v) OVA for $30 \mathrm{~min}$, three times a week, between days 21 and 63, using an ultrasonic nebulizer (Omron NE-U07; Omron, Kyoto, Japan). Subgroups of mice received a single i.p. injection of the Clara cell-specific cytotoxicant, NA $(200 \mathrm{mg} / \mathrm{kg}$ body weight; Sigma-Aldrich) (i) on day 20 (1 day before the first OVA nebulization period; NA + OVA group; $n=10$ ); (ii) on day 60 (during the second last OVA nebulization period; OVA/NA group; $n=10$ ); or (iii) on day 64 ( 1 day after the last OVA nebulization period; OVA + NA group; $n=12$; Figure 1).

Separate subgroups of mice subjected to the (iv) OVA-induced model of chronic AI-induced AAD alone $(n=10)$ or injected with (v) $0.5 \mathrm{ml}$ saline (vehicle control for OVA; $n=6$ ) and nebulized with saline instead of OVA (from days 21 to 63) were maintained until day 67 as controls of the combined model of AAD (Figure 1). Additionally, age-matched mice maintained under standard housing conditions until day 63 (to control for the period of time that OVA + NA-treated mice were being subjected to the 9-week OVA-induced model of AAD), and (vi) injected i.p. with NA $(n=10)$ or (vii) corn oil (vehicle control for NA; Sigma-Aldrich; $n=6$ ) on day 64 and maintained until day 67 acted as separate controls of the combined model of AAD (Figure 1).

\section{Invasive Plethysmography}

On day 67 , all seven groups of mice $(n=64$ in total) had their airway resistance (AHR) measured by invasive 


\section{i) COMBINED NA+OVA GROUP}

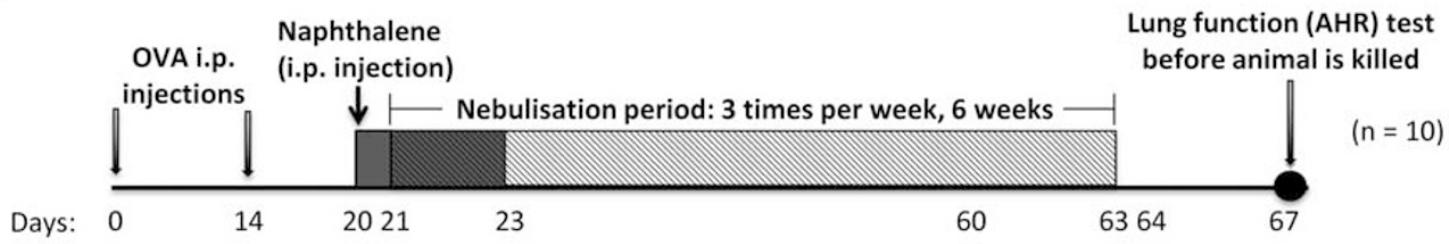

ii) COMBINED OVA/NA GROUP

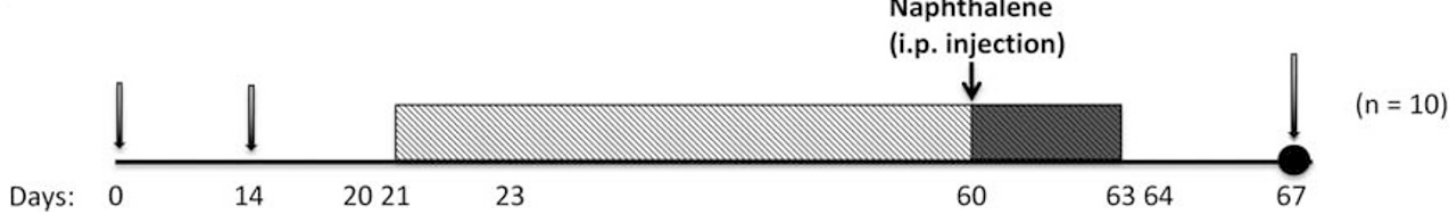

iii) COMBINED OVA+NA GROUP

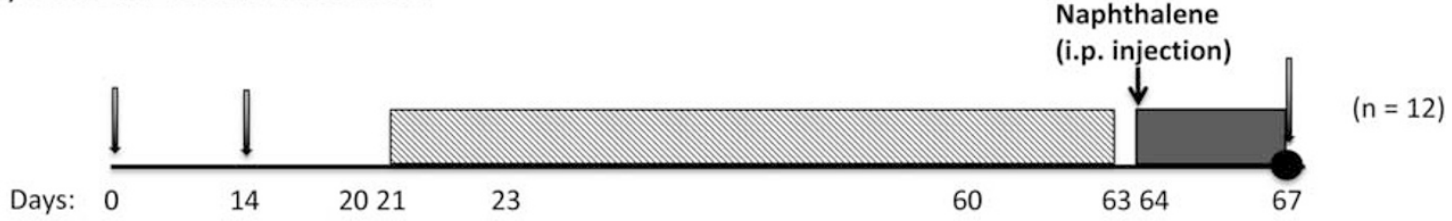

iv) OVA ALONE GROUP

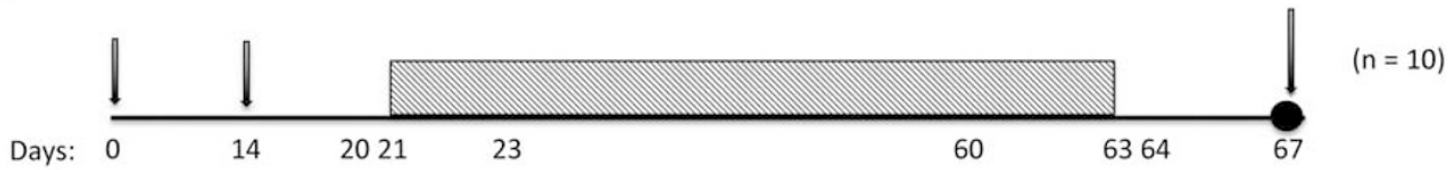

v) SALINE ALONE GROUP

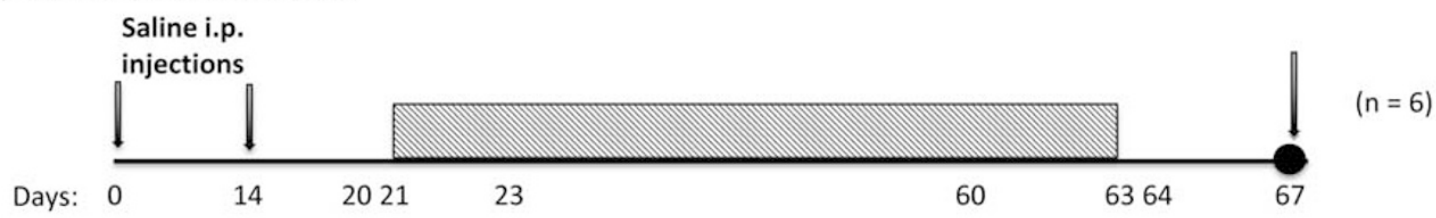

vi) NA ALONE GROUP

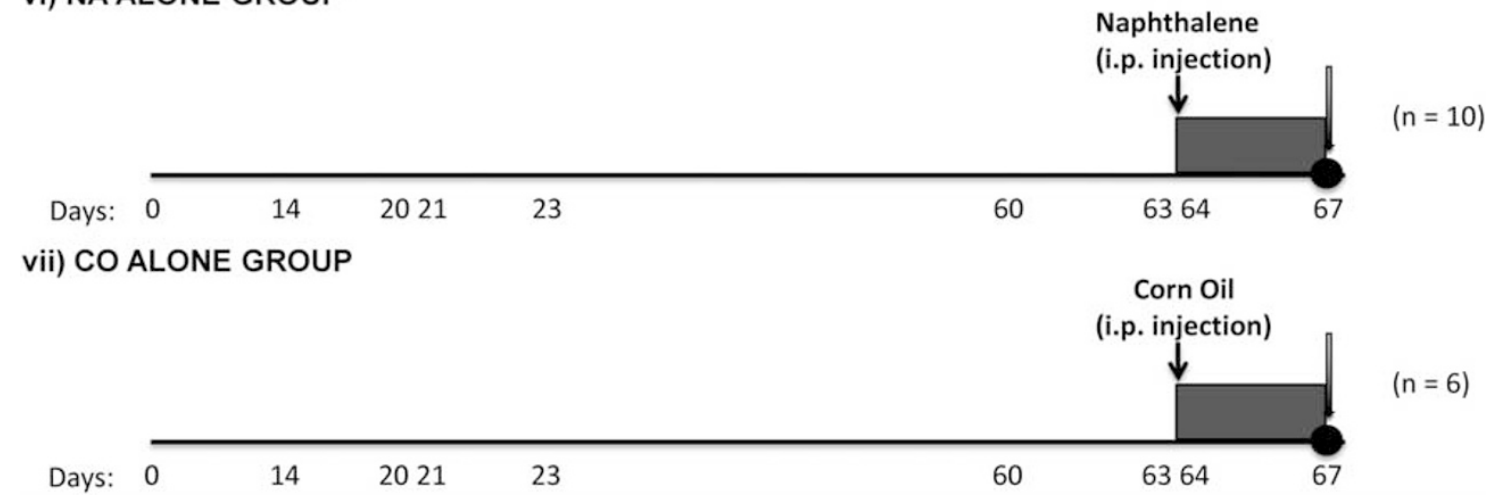

Figure 1 A schematic illustration of how the combined (i) NA + OVA, (ii) OVA/NA, and (iii) OVA + NA-treated models were established, along with separate control groups subjected to (iv) OVA-induced AAD or (v) saline (vehicle for OVA), and (vi) NA-induced epithelial damage or (vii) CO (vehicle for $\mathrm{NA}$ ). Note that the (vi) NA and (vii) CO alone groups were housed for 9 weeks prior to treatment, to control for the period of time that the combined groups (i-iii) were being subjected to the OVA-induced component of the model. 
plethysmography, in response to increasing concentrations of methacholine-induced airway bronchoconstriction. Briefly, mice were anesthetized with an i.p. injection of ketamine $(200 \mu \mathrm{g} / \mathrm{g})$ and xylazine $(10 \mu \mathrm{g} / \mathrm{g})$, tracheostomized and the jugular vein cannulated. Mice were then ventilated with a small animal respirator (Harvard Apparatus, Holliston, MA, USA) delivering $0.01 \mathrm{ml} / \mathrm{g}$ body weight at a rate of 120 strokes per minute in a mouse plethysmograph chamber. Increasing doses of methacholine were delivered i.v. and AHR was measured (Biosystem XA version 2.7.9, Buxco Electronics, Troy, NY, USA) for $2 \mathrm{~min}$ after each dose. Results were then expressed as the maximal resistance after each dose of methacholine minus baseline resistance.

\section{Bronchoalveolar Lavage (BAL)}

Three $0.5 \mathrm{ml}$ lavages were pooled in ice-cold $20 \%$ fetal calf serum (FCS)/phosphate-buffered saline (PBS) before red blood cells were lysed and cells washed twice in 5\% FCS/PBS. Total viable cell counts were performed manually by trypan blue exclusion. Cytospin smears $\left(2 \times 10^{4}\right.$ cells $)$ were prepared, fixed with methanol and stained with modified Wright's stain (Hema-Tek, Bayer Diagnostics, Leverkusen, Germany). Differential counts of eosinophils, neutrophils, lymphocytes and monocytes were determined using light microscopy $(\times 40$ magnification, 100 cells counted $)$ in a blinded manner.

\section{Tissue Collection}

Once airway reactivity measurements were completed, animals were killed with an overdose of anesthetic before their lung tissue isolated and rinsed in cold PBS. The lungs of each animal were then divided along the transverse plane, resulting in four separate lobes. The largest lobe was fixed in $10 \%$ neutral buffered formalin overnight, processed routinely and embedded in paraffin wax. The remaining three lobes were snap-frozen in liquid nitrogen and stored at $-80^{\circ} \mathrm{C}$ for further analyses, as detailed below.

\section{Lung Histopathology}

Formalin-fixed, paraffin-embedded tissues were sectioned ( $3 \mu \mathrm{m}$ thickness) and placed on SuperFrost charged microscope slides (Grale Scientific, Melbourne, VIC, Australia). To assess peri-bronchial inflammation score, one set of serial sections/mouse underwent Mayer's haematoxylin and eosin Y (H \& E) staining. To assess epithelial thickness, epithelial denudation and subepithelial collagen deposition, another set of serial sections/mouse underwent Masson trichrome staining. To assess goblet cell metaplasia, a third set of serial sections/mouse underwent Alcian Blue Periodic Acid Schiff (AB-PAS) staining.

\section{Histological Evaluation of Inflammation}

Sections stained with H\&E were observed under low power light microscopy ( $\times 40$ magnification). Histological grading of inflammation severity from 0 to 4 was assigned to every slide $(0=$ no detectable inflammation; $1=$ occasional inflammatory cell aggregates, pooled size $<0.1 \mathrm{~mm}^{2}$; $2=$ some inflammatory cell aggregates, pooled size $\sim 0.2 \mathrm{~mm}^{2} ; 3=$ widespread inflammatory cell aggregates, pooled size $\sim 0.3 \mathrm{~mm}^{2}$; and $4=$ widespread and massive inflammatory cell aggregates, pooled size $\sim 0.6 \mathrm{~mm}^{2}$ ), and was performed blinded by the same investigator. The area of inflammatory cell aggregates was measured using Image Pro Discovery software (Media Cybernetics, Silver Spring, MD, USA) in $\mu \mathrm{m}^{2}$ and converted to $\mathrm{mm}^{2}$.

\section{Immunohistochemistry}

Paraffin-embedded lung sections were immunohistochemically stained for annexin $\mathrm{V}$ (a marker of apoptosis) ${ }^{23}$ and TGF- $\beta 1^{23}$ as described before: utilizing polyclonal antibodies to annexin V (S0961; 1:100 dilution; Epitomics, Burlingame, CA, USA) and TGF- $\beta 1$ (sc146; 1:800 dilution; Santa Cruz Biotechnology, Santa Cruz, CA, USA), respectively. Detection of antibody staining was completed using the Dako EnVision anti-rabbit kit (Dako, Carpinteria, CA, USA) and 3,3'-diaminobenzidine (Sigma-Aldrich), where sections were counterstained with hematoxylin. Images of five bronchi (measuring 150-350 $\mu \mathrm{m}$ luminal diameter) per section were obtained and quantified by morphometry, as described below.

\section{Morphometric Analysis}

Representative photomicrographs from Masson trichromeand AB-PAS-stained slides were captured from scanned images using ScanScope AT Turbo (Aperio, CA, USA), while immunohistochemically stained images were captured using an Olympus UC50 camera (Olympus Australia, Melbourne, VIC, Australia) attached to an Olympus BX51 microscope. Stained airways were randomly selected from across the tissue sample. Masson trichrome-stained slides were analyzed by measuring the thickness of the epithelial and subepithelial layers and expressing the value as $\mu \mathrm{m}^{2} / \mu \mathrm{m}$ basement membrane length. AB-PAS slides were analyzed by counting the number of stained goblet cells, which were expressed as the number of goblet cells $/ 100 \mu \mathrm{m}$ basement membrane length. Annexin V- and TGF- $\beta 1$-stained sections were analyzed by scoring the degree of staining around the membrane of the airway epithelial cells between 0 and 3 (where 0 represented no staining and 3 represented abundant staining).

\section{Hydroxyproline Assay}

The second largest lung lobe from each mouse was processed as described before ${ }^{11,23}$ for the measurement of hydroxyproline content, which was determined from a standard curve of purified trans-4-hydroxy-L-proline (Sigma-Aldrich). Hydroxyproline values were multiplied by a factor of 6.94 (based on hydroxyproline representing $\sim 14.4 \%$ of the amino-acid composition of collagen in most mammalian 

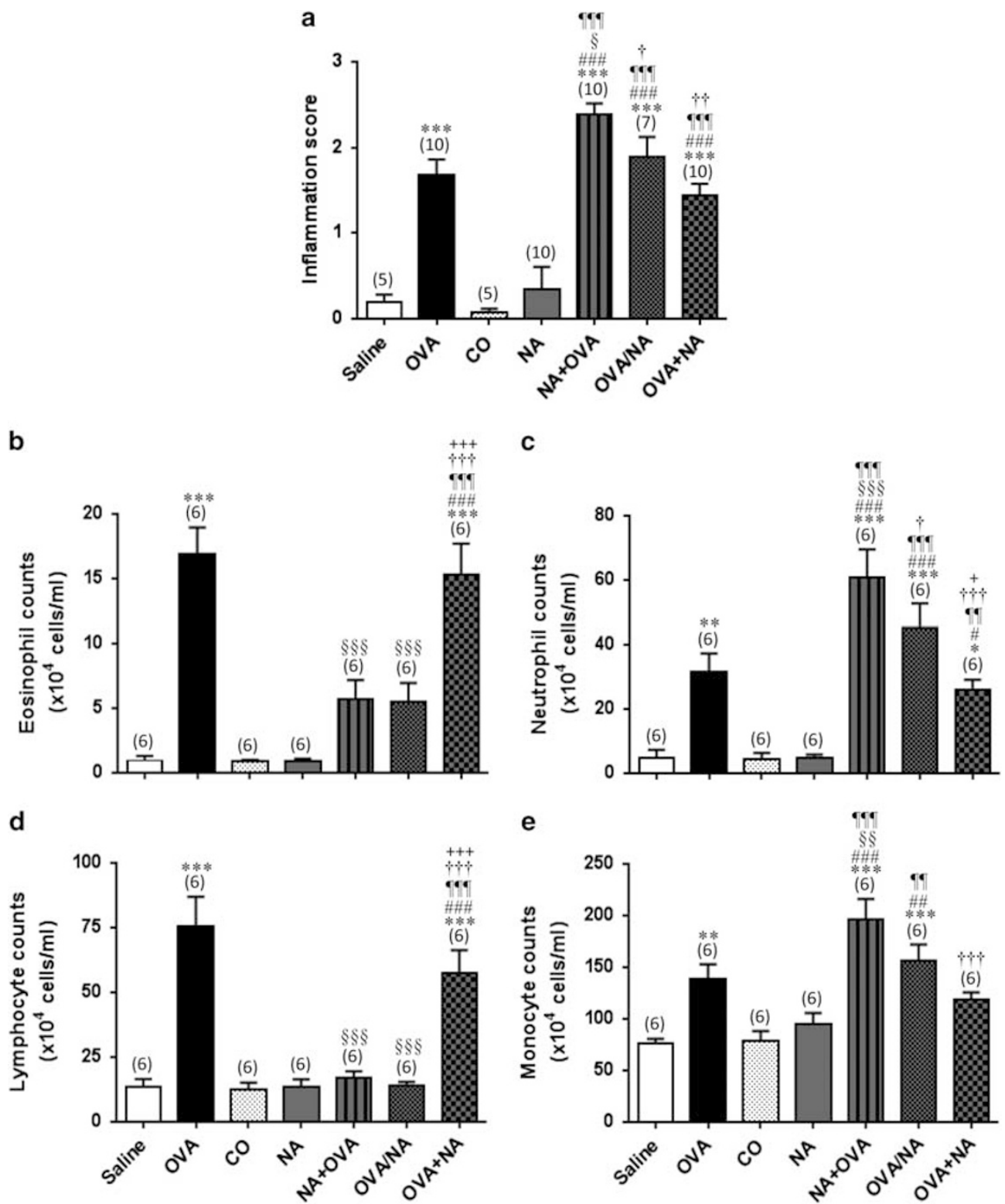

Figure 2 Determination of airway inflammation score from H\&E-stained airways and BAL differential cell counts. The mean \pm s.e.m. (a) inflammation score in H\&E-stained lung sections, and number of (b) eosinophils, (c) neutrophils, (d) lymphocytes, and (e) monocytes in BAL fluid from salinesensitized/challenged (saline), OVA-sensitized/challenged (OVA), CO-treated (CO), NA-treated (NA), and combined-treated mice. Numbers in parenthesis represent the number of animals analyzed per group. ${ }^{*} P<0.05,{ }^{*} P<0.01,{ }^{* *} P<0.001$ vs saline group; ${ }^{\#} P<0.05,{ }^{\# \#} P<0.01,{ }^{\# \# \#} P<0.001$ vs CO group;

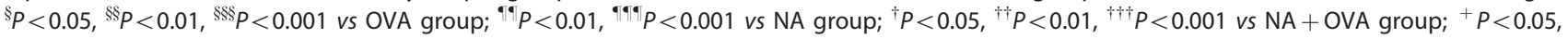
$+++P<0.001$ vs OVA/NA group.

Figure 3 Determination of airway epithelial denudation, epithelial thickness and subepithelial collagen thickness from Masson trichrome-stained airways. (a) Representative photomicrographs of lung sections from saline-sensitized/challenged (saline), OVA-sensitized/challenged (OVA), CO-treated (CO), NA-treated (NA), and combined-treated mice, stained with Masson trichrome. The mean \pm s.e.m. (b) epithelial denudation (expressed as a \% relative to BM length; indicated by arrows in the representative image of NA-treated mice), (c) epithelial thickness (in mm, relative to BM length), and (d) subepithelial collagen thickness (expressed in $\mu \mathrm{m}$ relative to BM length) were then morphometrically assessed from Masson trichrome-stained sections derived from each animal analyzed. Also shown is the mean \pm s.e.m. total collagen concentration (\% collagen content per dry weight tissue), which was extrapolated from corresponding hydroxyproline values. Numbers in parentheses represent the number of animals analyzed per group.

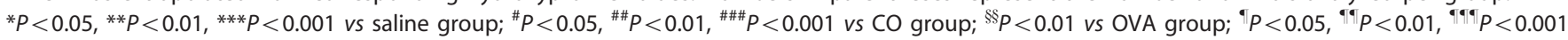
vs NA group; ${ }^{\dagger} P<0.05$ vs NA + OVA group; ${ }^{++}+P<0.001$ vs OVA/NA group. 
tissues), ${ }^{24}$ to extrapolate total collagen content, which in turn was divided by the dry weight of each corresponding tissue to yield percent collagen concentration.

\section{Statistical Analysis}

All data are expressed as the mean + s.e.m. Lung function studies were analyzed by a two-way ANOVA with Bonferroni
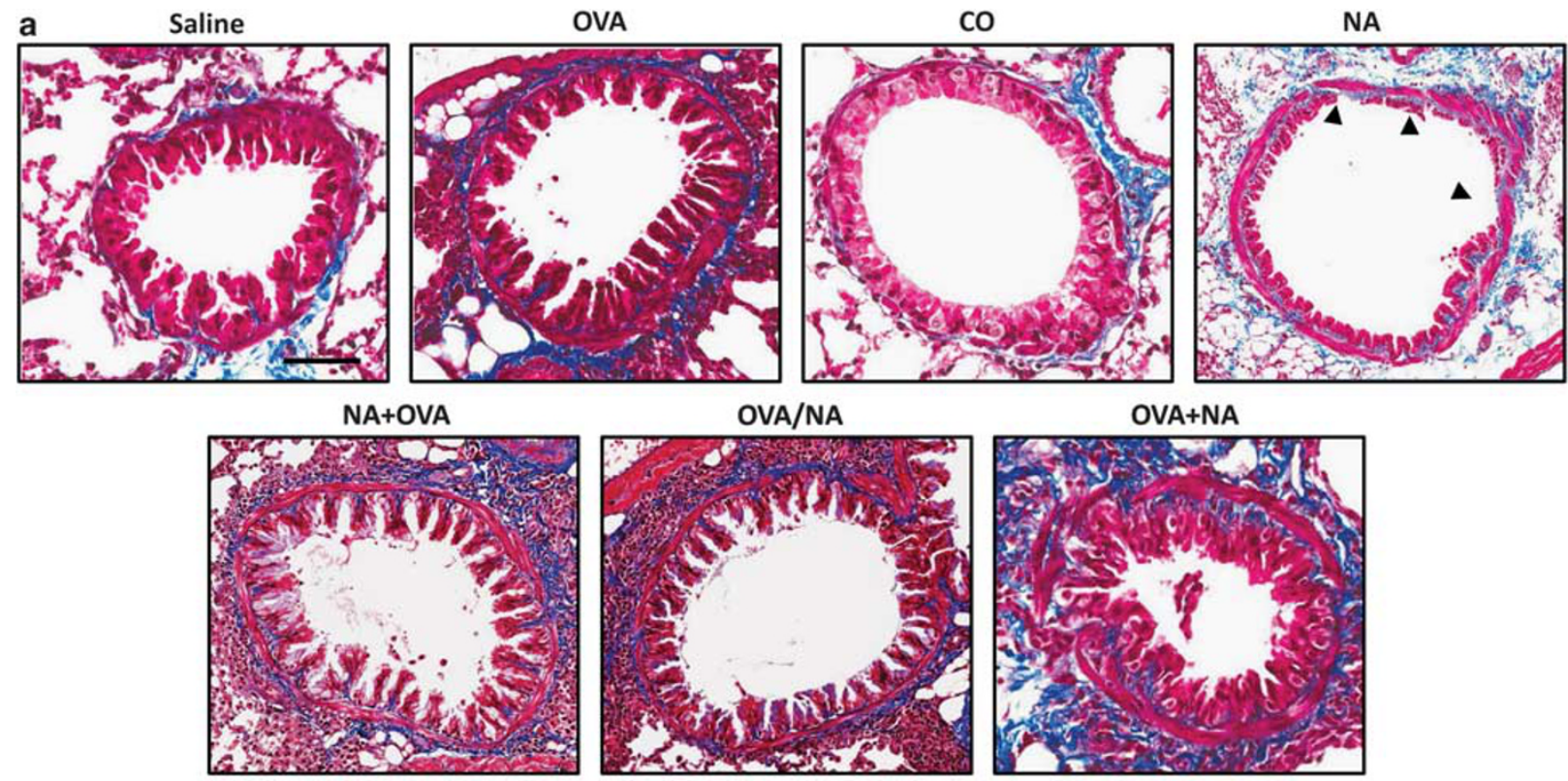

b
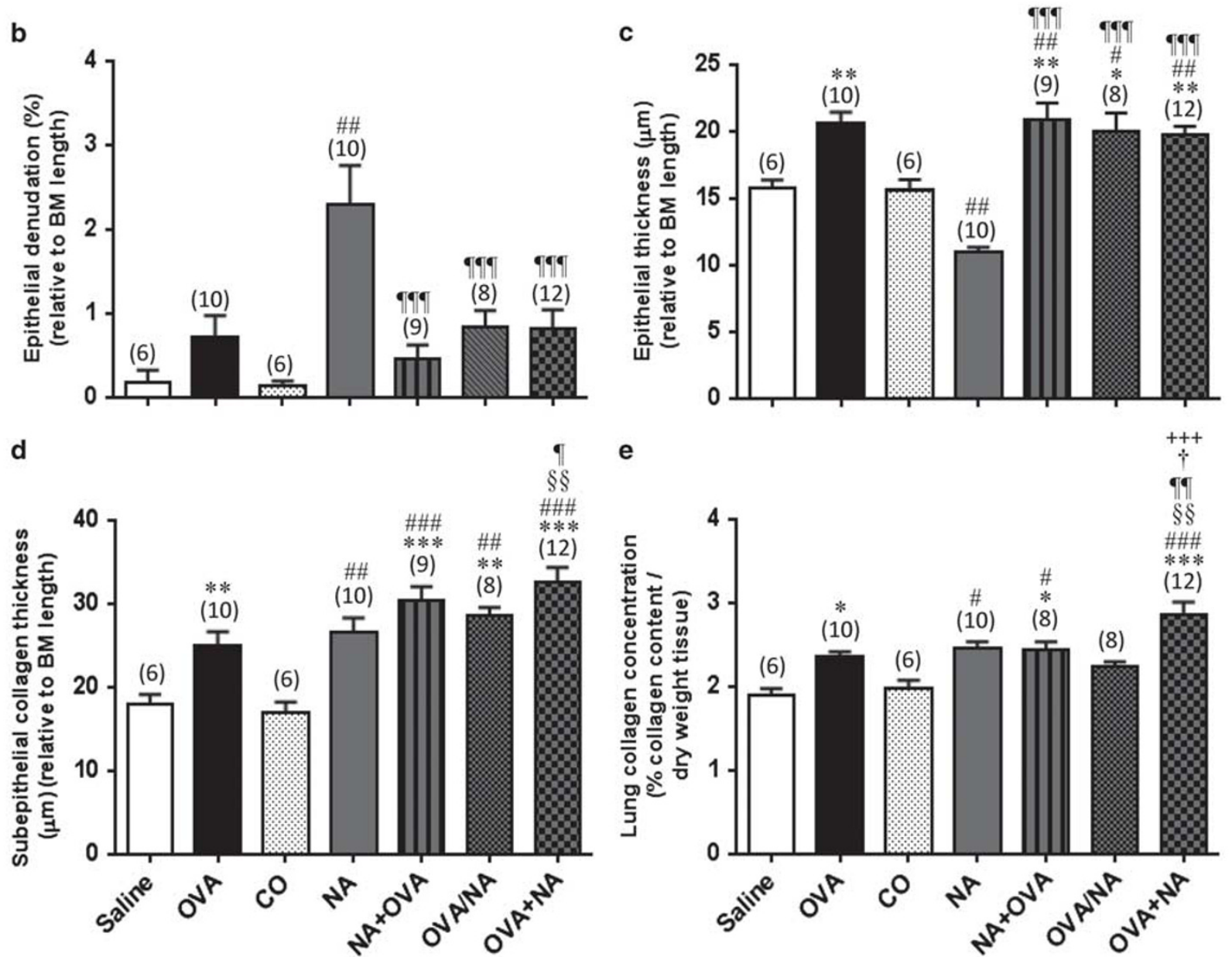
post hoc test, whereas the remaining data were analyzed by a one-way ANOVA with Neuman-Keuls post hoc test for multiple comparisons between groups. In each case, significance was classified as being $P<0.05$.

\section{RESULTS}

\section{Individual vs Combined Effects of OVA-Induced AAD and NA-Induced Epithelial Damage on AI}

Confirmation that OVA-treated mice were adequately sensitized/challenged was demonstrated by an increased inflammation score in these animals compared with that measured in saline-treated counterparts (OVA: $1.68 \pm 0.18 \mathrm{vs}$ saline: $0.20 \pm 0.08 ; P<0.001$ vs saline group; Figure 2a). Consistent with this, OVA-treated mice had significantly increased eosinophils (Figure 2b), neutrophils (Figure 2c), lymophocytes (Figure 2d), and monocytes (Figure 2e) in their BAL fluid compared with respective measurements obtained from saline-treated mice (all $P<0.01 v s$ saline group). On the other hand, inflammation score and differential BAL counts were not significantly different between NA- vs CO-treated mice (Figure 2). Interestingly, NA + OVA-treated mice had a higher inflammation score than that measured in OVA-sensitized/challenged animals (NA + OVA: $2.38 \pm 0.14$ vs OVA: $1.68 \pm 0.18 ; P<0.05$ vs OVA group; Figure 2a). This was associated with significantly higher neutrophils (Figure $2 c$ ) and monocytes (Figure 2e; both $P<0.01$ vs respective measurements from the OVA group) in the BAL of these mice, while eosinophil (Figure 2b) and lymphocyte (Figure 2d) counts in $\mathrm{NA}+$ OVA-treated mice were significantly lower than the respective measurements obtained from their OVA alonetreated counterparts. OVA/NA-treated mice had a comparable inflammation score $(1.89 \pm 0.23$; Figure $2 \mathrm{a})$ as well as BAL neutrophil (Figure 2c) and monocyte (Figure 2e) counts, but significantly lower BAL eosinophils (Figure 2b) and lymphocytes than those measured in OVA alone-treated mice. Only OVA + NA-treated mice had a comparable inflammation score (1.44 \pm 0.14 ; Figure 2a) and differential BAL counts (Figure $2 \mathrm{~b}-\mathrm{e}$ ) to that measured in OVA-sensitized/challenged mice.

\section{Individual vs Combined Effects of OVA-Induced AAD and NA-Induced Epithelial Damage on Features of Epithelial Remodeling}

Epithelial denudation

Epithelial denudation (extent of epithelial cell loss per $100 \mu \mathrm{m}$ of basement membrane) was assessed by morphometric analysis of Masson trichrome-stained lung tissue sections (Figure 3a). The mean denudation was not significantly different in OVA-sensitized/challenged mice compared with that from saline-sensitized/challenged animals (OVA: $0.72 \pm 0.25$ vs saline: $0.19 \pm 0.14$ ). Conversely, epithelial denudation in NA-treated mice was significantly increased compared with that measured from CO-treated mice (NA: $2.31 \pm 0.45$ vs CO: $0.13 \pm 0.06 ; P<0.001$ vs CO group). In comparison, epithelial denudation in $\mathrm{NA}+\mathrm{OVA}$
$(0.46 \pm 0.17), \quad$ OVA/NA $\quad(0.84 \pm 0.20), \quad$ and $\quad \mathrm{OVA}+\mathrm{NA}$ $(0.83 \pm 0.22)$ treated mice was comparable with that measured in OVA-sensitized/challenged mice $(0.72 \pm 0.25)$, but was significantly lower than that measured in NA-treated animals $(2.31 \pm 0.45$; all $P<0.001 v s$ the NA group; Figure $3 b)$.

\section{Epithelial thickness}

Airway epithelial thickness was also assessed from Masson trichrome-stained lung tissue sections (Figure 3a) and was significantly increased in OVA-sensitized/challenged mice compared with that in saline-sensitized/challenged control animals (OVA: $20.58 \pm 0.86$ vs saline: $15.74 \pm 0.62 ; P<0.01$ vs saline group). Conversely, epithelial thickness in NA-treated mice was significantly decreased than that measured from CO-treated mice (NA: $10.94 \pm 0.43$ vs CO: $15.64 \pm 0.75$; $P<0.01$ vs CO group). In comparison, epithelial thickening in NA+ OVA $(20.90 \pm 1.25)$, OVA/NA $(19.99 \pm 1.41)$ and OVA + NA (19.74 \pm 0.65$)$ treated mice was comparable to that measured in OVA-sensitized/challenged animals (20.58 \pm 0.86$)$, but was significantly higher than that measured in NA-treated mice (10.94 \pm 0.43 ; all $P<0.001$ vs NA group; Figure 3c).

\section{Goblet cell metaplasia}

Goblet cell metaplasia (number of goblet cells/100 $\mu \mathrm{m}$ basement membrane length) was assessed by morphometric analysis of AB-PAS-stained lung tissue sections (Figure 4a). The mean number of goblet cells was significantly increased in OVA-sensitized/challenged mice compared with that from saline-sensitized/challenged animals (OVA: $6.02 \pm 0.59$ vs saline: $0.13 \pm 0.05 ; P<0.001$ vs saline group; Figure $4 \mathrm{~b}$ ). Conversely, there was no significant change in mean goblet cell numbers between NA-treated and CO-treated groups (NA: $0.45 \pm 0.14$ vs CO: $0.01 \pm 0.01$; Figure $4 \mathrm{~b}$ ). In comparison, goblet cell numbers in NA+OVA-treated mice ( $4.83 \pm 0.57 ; P<0.001$ vs saline, CO and NA alone groups) were not significantly different from that measured in OVAsensitized/challenged mice $(6.02 \pm 0.59)$, but progressively decreased in OVA/NA $(4.10 \pm 0.81 ; P<0.05$ vs OVA group; $P<0.01$ vs saline group; $P<0.001$ vs $\mathrm{CO}$ and NA alone groups), and OVA + NA $(2.82 \pm 0.52 ; P<0.01$ vs OVA alone, $\mathrm{CO}$ and NA alone groups; $P<0.05 v s$ saline and NA + OVA groups) treated animals (Figure $4 \mathrm{~b}$ ).

\section{Individual vs Combined Effects of OVA-Induced AAD and NA-Induced Epithelial Damage on Airway Fibrosis Subepithelial lung collagen thickening}

Subepithelial collagen thickness (relative to basement membrane length) was assessed from Masson trichrome-stained lung tissue sections (Figure 3a), was significantly increased in OVA-sensitized/challenged mice compared with that from saline-treated mice (OVA: $24.95 \pm 1.70$ vs saline: $17.99 \pm 1.19$; $P<0.01$ vs saline group), and separately in NA-treated mice compared with CO-treated control mice (NA: $26.61 \pm 1.70 \mathrm{vs}$ CO: $17.0 \pm 1.26 ; P<0.01$ vs CO group; Figure 3c). Sub- 
epithelial collagen thickness in NA + OVA (30.46 \pm 1.60$)$ and OVA/NA $(28.58 \pm 0.99)$ treated mice were slightly higher but not statistically different from that in OVA alone $(24.95 \pm 1.70)$ and NA alone $(26.61 \pm 1.70)$ injured mice (both $P<0.01$ vs saline and CO groups; Figure 3c). Strikingly however, subepithelial collagen thickness in OVA + NAtreated mice $(32.61 \pm 1.78)$ was further increased such that it was significantly greater than that measured in OVA $(P<0.01$ vs OVA group) and NA $(P<0.05$ vs NA group) treated mice (Figure 3c).

\section{Total lung collagen concentration}

Total lung collagen concentration (percentage of collagen content/dry weight lung tissue) was extrapolated from hydroxyproline analysis (Figure 3d), significantly increased in OVA-sensitized/challenged mice compared with that measured from saline-sensitized/challenged control mice (OVA: $2.35 \pm 0.07$ vs saline: $1.90 \pm 0.07 ; P<0.05$ vs saline group), and separately in NA-treated mice compared with CO-treated mice (NA: $2.47 \pm 0.07$ vs CO: $1.98 \pm 0.10 ; P<0.05$ vs $\mathrm{CO}$ group; Figure $3 \mathrm{~d}$ ). In a similar trend to changes in subepithelial collagen thickness, total lung collagen concentration in NA + OVA $(2.45 \pm 0.08 ; P<0.05$ vs saline and CO groups) and OVA/NA $(2.25 \pm 0.05)$ treated mice was not statistically different from that in OVA alone $(2.35 \pm 0.07)$ and NA alone $(2.47 \pm 0.07)$ injured mice, whereas collagen concentration in OVA + NA-treated animals $(2.86 \pm 0.15)$ was significantly greater than that measured in all other groups $(P<0.001$ vs saline, $\mathrm{CO}$ and OVA/NA groups; $P<0.01$ vs OVA alone and NA alone groups; $P<0.05$ vs $\mathrm{NA}+$ OVA group; Figure 3d).

\section{Individual vs Combined Effects of OVA-Induced AAD and NA-Induced Epithelial Damage on AHR}

Changes in AHR from baseline in response to increasing doses of nebulized methacholine were used to measure changes in AHR between treatment groups (Figure 5). There was a significant increase in AHR in OVA-sensitized/challenged mice compared with that measured from saline-sensitized/challenged controls $(P<0.05$ vs saline group $)$. In contrast, there was no significant difference in airway reactivity between NA-treated and CO-treated mice, consistent with previous studies ${ }^{23}$ and reflecting the fact that NA-treated mice undergo reparative healing by 3 days post injury, which is not associated with increased AHR. In comparison, only OVA + NA-treated mice exhibited significantly enhanced AHR compared with that measured from OVA-sensitized/challenged ( $P<0.05 v s$ OVA group) and NA-treated mice $(P<0.001$ vs NA group; Figure 5$)$.

\section{Individual vs Combined Effects of OVA-Induced AAD and NA-Induced Epithelial Damage on Other Parameters of Epithelial Damage and Fibrosis}

Based on the findings detailed above, changes in airway epithelium apoptosis and the pro-fibrotic factor, transform- ing growth factor (TGF)- $\beta 1$, were additionally evaluated in OVA + NA-treated mice and compared with that in the four control groups:

\section{Epithelium apoptosis}

Epithelial cell apoptosis was assessed by morphometric analysis of annexin V-stained lung tissue sections (Figure 6a). Annexin $\mathrm{V}$ staining was significantly increased in OVAsensitized/challenged mice compared with that from saline-sensitized/challenged animals (OVA: $1.51 \pm 0.19$ vs saline: $0.76 \pm 0.17 ; P<0.01$ vs saline group) and separately in NA-treated mice compared with that measured from COtreated mice (NA: $2.17 \pm 0.12 v s \mathrm{CO}: 0.40 \pm 0.10 ; P<0.001$ vs $\mathrm{CO}$ group; Figure $6 \mathrm{~b})$. In comparison, OVA + NA-treated mice had significantly increased levels of annexin $\mathrm{V}$ staining compared with that from OVA-sensitized/challenged mice (OVA + NA: $1.90 \pm 0.07 v s$ OVA: $1.51 \pm 0.19 ; P<0.05 v s$ OVA group), but comparable levels of apoptosis to that measured from NA-treated animals (OVA + NA: $1.90 \pm 0.07$ vs NA: $2.17 \pm 0.12$ Figure $6 \mathrm{~b}$ ).

\section{TGF- $\beta 1$ expression}

TGF- $\beta 1$ expression/distribution was assessed by morphometric analysis of TGF- $\beta 1$-stained lung tissue sections (Figure $6 \mathrm{c}$ ). TGF- $\beta 1$ staining was significantly increased in OVA-sensitized/challenged mice compared with that from saline-sensitized/challenged animals (OVA: $2.36 \pm 0.26$ vs saline: $1.00 \pm 0.15 ; P<0.001$ vs saline group) and separately in NA-treated mice compared with that measured from COtreated animals (NA: $2.07 \pm 0.73$ vs CO: $1.07 \pm 0.35 ; P<0.01$ vs $\mathrm{CO}$ group; Figure $6 \mathrm{~d}$ ). Mean airway TGF- $\beta 1$ staining levels trended to be higher in OVA + NA-treated mice $(2.71 \pm 0.15)$ compared with that in OVA alone $(2.36 \pm 0.26)$ and NA alone $(2.07 \pm 0.73)$ treated animals, although this did not reach statistical significance (Figure 6d).

\section{DISCUSSION}

Epithelial damage has emerged as a novel etiology of asthma, contributing to the development of AWR and subsequently AHR. ${ }^{23,25}$ Despite its significance, epithelial damage has been poorly addressed in current experimental models used to study the pathogenesis of asthma, and also by currently available therapies for asthma. Thus, establishing experimental models of $\mathrm{AAD}$ that better reflect the pathogenesis of human asthma and incorporate the contribution of epithelial damage with other features of AWR and AHR is warranted. The current study, therefore, aimed to superimpose epithelial damage at varying time points onto the 'gold-standard' model of chronic AAD (that presents with AI, AWR, and AHR) in mice, and determined how closely these models presented with features of human asthma. The main findings obtained were that (1) airway fibrosis and AHR induced by superimposing epithelial damage after AI-induced AWR (in the OVA + NA-treated group) were further exacerbated and significantly increased 
a

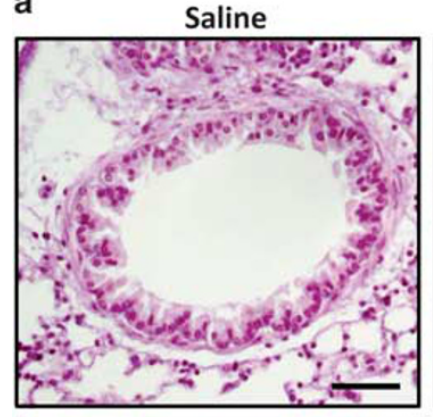

OVA

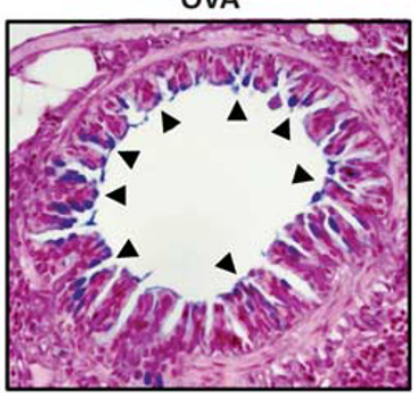

CO

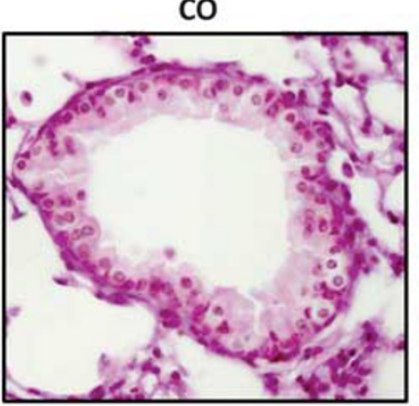

NA

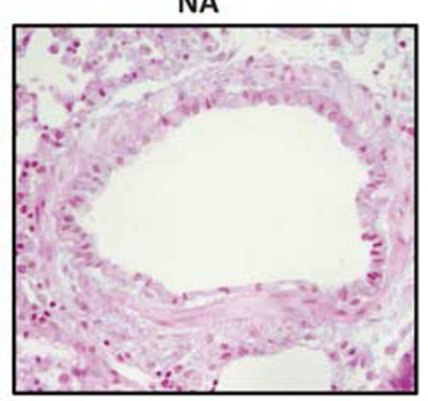

OVA+NA
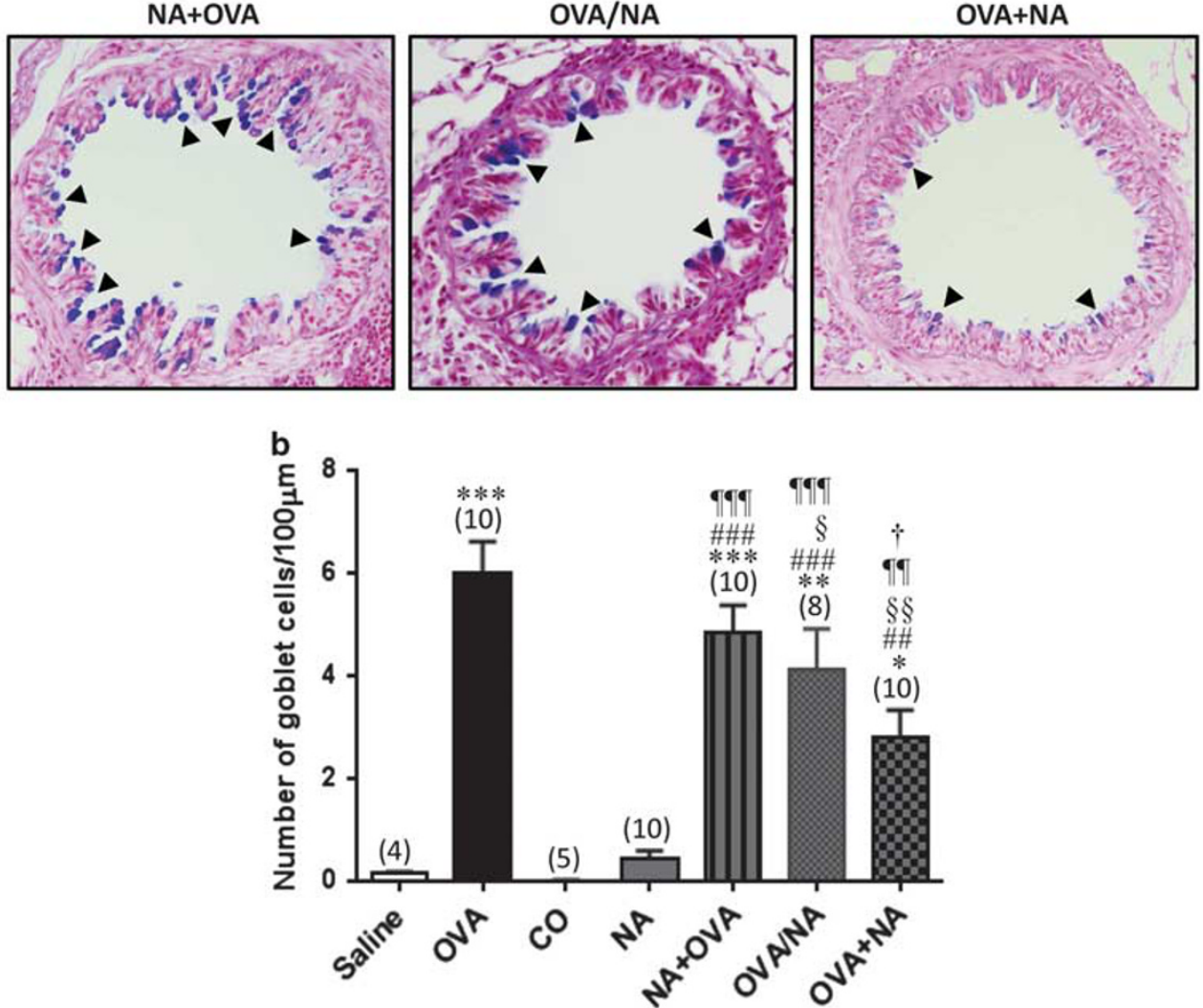

Figure 4 Determination of goblet cell number from Alcian blue periodic acid Schiff (AB-PAS) stained-airways. (a) Representative photomicrographs of lung sections from saline-sensitized/challenged (Saline), OVA-sensitized/challenged (OVA), CO-treated (CO), NA-treated (NA), and and combined-treated mice, stained with AB-PAS. The relative mean \pm s.e.m. (b) goblet cell count (number of goblet cells $/ 100 \mu \mathrm{m}$; indicated by arrows in the representative image of OVA and combined-treated mice) was then morphometrically assessed from AB-PAS-stained sections derived from each animal analyzed. Numbers in parentheses represent the number of animals analyzed per group. ${ }^{*} P<0.05,{ }^{* *} P<0.01,{ }^{* * *} P<0.001$ vs saline group; ${ }^{\# \#} P<0.01$, ${ }^{\# \# \#} P<0.001$

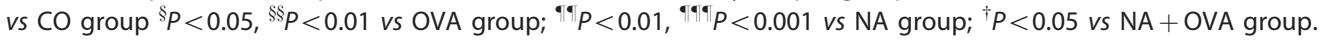

compared with that induced by AI alone (in the OVAsensitized/challenged group) or epithelial damage alone (in the NA-treated group); and (2) AI and related AWR-induced bronchial epithelium apoptosis (in the OVA-sensitized/ challenged group) was further exacerbated by epithelial damage (in the OVA + NA-treated group). However, (3) while epithelial damage in isolation (in the NA-treated group) led to a marked increase in epithelial denudation and significant reduction in epithelial thickness (compared with that measured in CO-treated control mice), it did not further affect these measures (in the NA+OVA, OVA/NA and
OVA + NA-treated groups) over and above that induced by AI-induced AWR (in the OVA-sensitized/challenged group). Additionally, (4) superimposing epithelial damage onto AI-induced AWR (in the combined models) resulted in a time-dependent progressive reduction in airway goblet cell metaplasia compared with that measured in OVA-sensitized/ challenged mice, with the lowest levels of goblet cells observed when epithelial damage was induced after AIinduced AWR (in the OVA + NA group).

From studies performed on the NA-induced model of epithelial damage and in mice deficient of the epithelial 


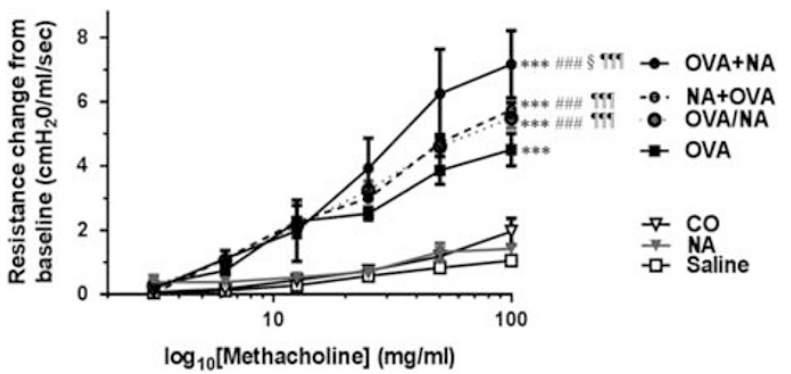

Figure 5 Determination of airway resistance from dose-response to methacholine. The relative mean \pm s.e.m. of resistance change from baseline $\left(\mathrm{cmH}_{2} \mathrm{O} / \mathrm{ml} / \mathrm{s}\right)$ was analyzed by conducting dose-responses to methacholine (a bronchoconstrictor) in saline-sensitized/challenged, OVAsensitized/challenged, CO-treated, NA-treated, and combined-treated mice. ${ }^{* *} P<0.001$ vs saline group; ${ }^{\# \# \#} P<0.01$ vs CO group; ${ }^{\S} P<0.05$ vs OVA group; $\because P<0.001$ vs NA group.

repair peptide, trefoil factor $2,{ }^{23,25}$ it can be suggested that epithelial damage alone can lead to subepithelial airway fibrosis (as demonstrated by the increased NA-induced subepithelial collagen thickness and total lung collagen concentration), similar to processes occurring in human asthma where repeated episodes of epithelial injury lead to prolonged activation of the epithelial mesenchymal trophic unit. ${ }^{26,27}$ Previous studies have shown that epithelial damage results in epithelial cells releasing pro-inflammatory factors such as IL-1 and tumor necrosis factor- $\alpha^{28}$ These factors in turn recruit and stimulate mast cells to release various factors including IL-10. IL-10 then actively recruits Th2 cells, which are stimulated to release IL-13, which in turn promotes the proliferation and differentiation of myofibroblasts from fibroblasts. ${ }^{3,28}$ IL-13 also stimulates the release of the profibrotic factor TGF- $\beta 1$ from myofibroblasts, ${ }^{3,28}$ which, when activated, stimulates the synthesis and deposition of increased matrix molecules in the subepithelial basement membrane region of the airway (resulting in increased subepithelial collagen thickness ${ }^{3,28,29}$ ) (Figure 7).

Additionally, the results of this study suggested that epithelial damage-induced fibrosis is further worsened when inflicted after the establishment of AI-induced AWR, leading to a concomitant worsening of AHR. Hence, both AI and epithelial damage can both independently cause inflammatory cell influx/activation and the release of various cytokines (IL-4, IL-10, IL-13, TGF- $\beta 1$ ) that result in myofibroblast differentiation and collagen secretion/deposition, and, when combined, result in an exacerbated level of fibrosis and related AHR (as demonstrated in the OVA + NA-treated group).

The findings from these studies also suggest that the degree of fibrosis is related to AHR, consistent with past studies ${ }^{30-32}$ that have shown that in addition to collagen IV being increased in the subepithelial layer various interstitial collagens are also synthesized and deposited into areas surrounding the subepithelial and within interstitial regions of the airway in human asthma. This increase in aberrant collagen deposition increases total wall thickness of the airway, resulting in a reduction in the diameter of the airway lumen. ${ }^{33,34}$ This feature can lead to an increase in AHR, which has been extensively mathematically modeled. ${ }^{33,34}$

While this exaggerated fibrosis and AHR observed in the OVA + NA-treated model is more likely to mimic the sequence of events that take place during chronic asthma, the alterations in some aspects of the epithelial remodeling changes observed (in OVA + NA-treated mice; such as epithelial denudation and goblet cell metaplasia) were less prominent. As expected, epithelial denudation was significantly increased by NA treatment of mice (as demonstrated previously). ${ }^{23,35}$ Comparatively though, epithelial denudation in OVA + NAtreated mice was similar to that measured OVA-sensitized/ challenged animals, but significantly less than in NA alonetreated mice. OVA sensitization/challenge stimulates a cascade of effects that leads to the differentiation of Clara cells (the predominant cell type in the mouse airway epithelium) into goblet cells, ${ }^{36,37}$ resulting in goblet cell metaplasia. Hence, by the time NA was administered to the combined model, less Clara cells may have been present (for the NA to target), correlating with the smaller amount of epithelial denudation that was observed in the OVA + NA-treated model. These findings potentially highlight the limitations of using isolated animal models of epithelial damage to mimic the pathology of human asthma, as the specific and exaggerated level of airway damage they undergo is likely to be higher than that associated with human disease. However, these findings also highlight that the etiology of epithelial damage is quite different in the combined murine model established from what occurs in humans, in which epithelial shedding is caused by an inherited predisposition to epithelial damage, which may include abnormal expression of cell-cell adhesion molecules. ${ }^{8}$ Furthermore, the histology of the human airway is more complex than that of the mouse with different cell renewal lineages. Despite this, the OVA + NA-treated model still results in pathology and an end point that resembles the human epithelial lesion. Further work in this combined mouse model including time point studies and repeated epithelial injury may reveal more about the chronic asthma scenario representative of the lifelong asthma sufferer. Furthermore, this model may enable evaluation of the effect of drugs on wound healing, which has only been characterized in laboratory animals, ${ }^{38}$ in the background of chronic inflammation and remodeling.

Expectedly, bronchial epithelium apoptosis was significantly higher in NA alone-treated mice (compared with that in CO-treated controls), ${ }^{23}$ consistent with the epithelial denudation that these mice exhibited. Annexin V-associated apoptosis was also significantly higher in OVA-sensitized/ challenged mice compared to that measured in saline controls, ${ }^{25}$ and was further increased in OVA + NA-treated mice compared with OVA-sensitized/challenged animals, which was comparable to levels measured in NA alonetreated mice. As epithelial denudation was not observed in 

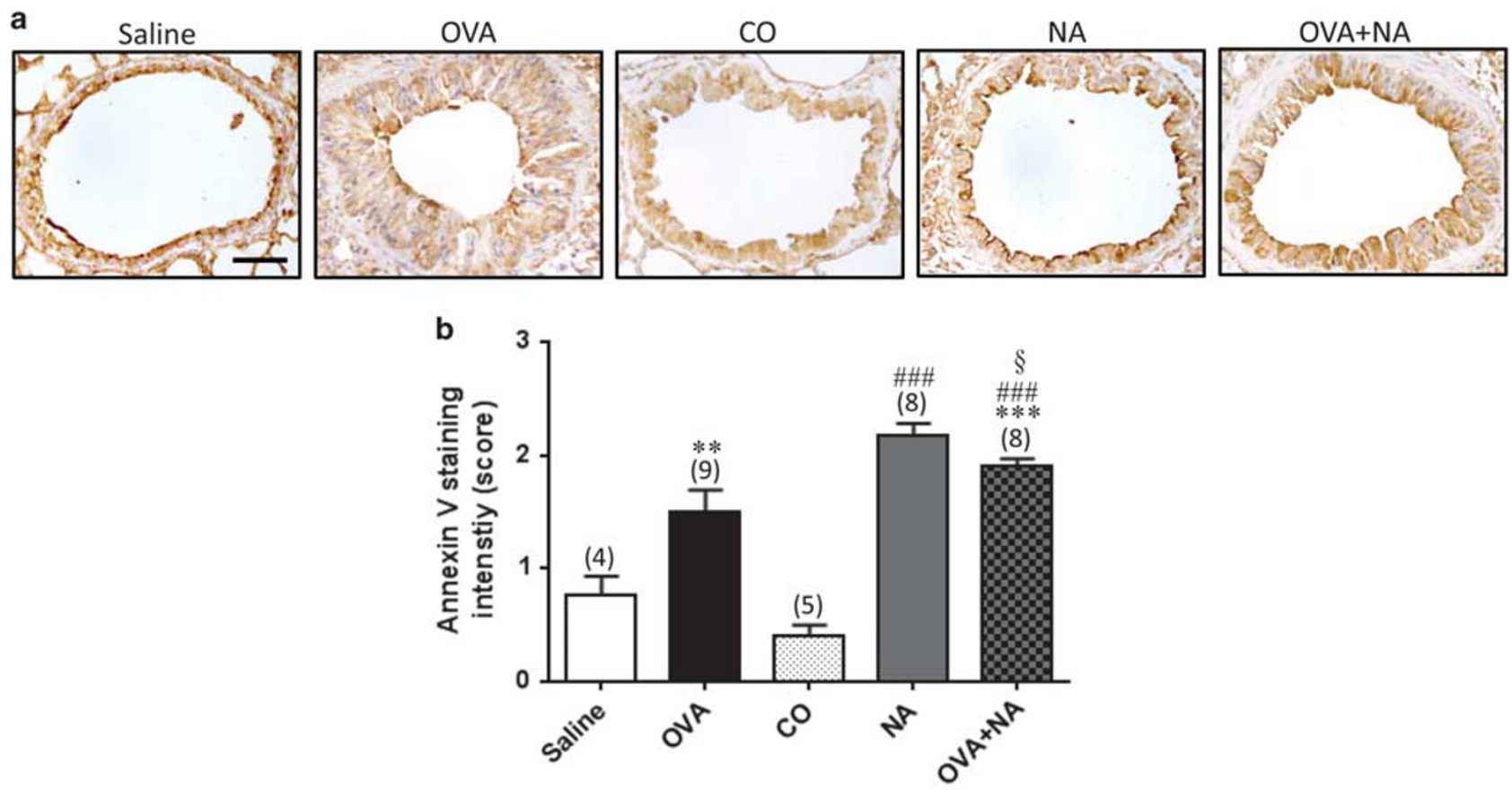

C

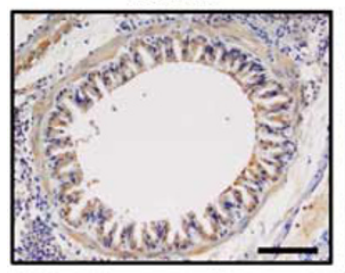

OVA

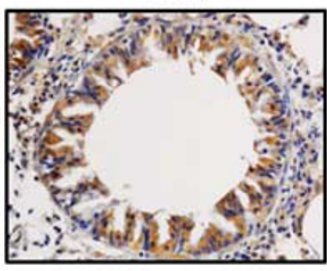

$\mathrm{CO}$

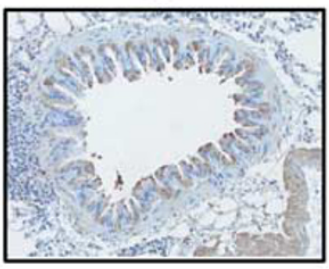

NA

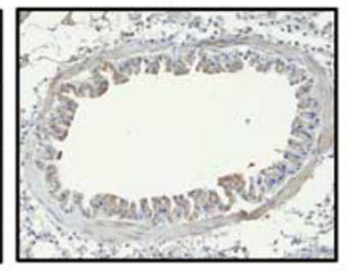

OVA+NA

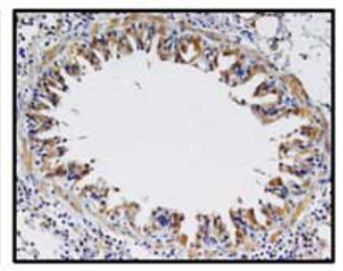

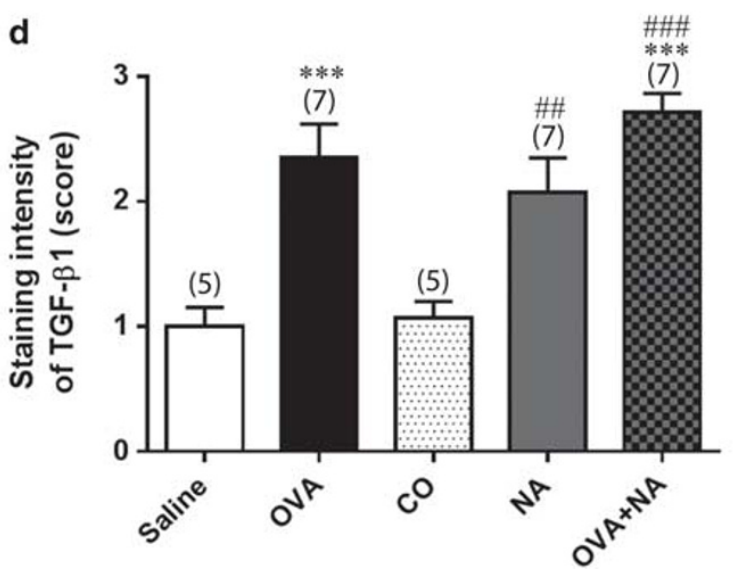

Figure 6 Determination of epithelial cell apoptosis and TGF- $\beta 1$ expression from immunohistochemically stained airways. Representative photomicrographs of lung sections from saline-sensitized/challenged (saline), OVA-sensitized/challenged (OVA), CO-treated (CO), NA-treated (NA), and combined-treated (OVA + NA) mice, stained for (a) annexin $V$ (a marker of apoptosis), and (c) TGF- $\beta 1$ expression (as a pro-fibrotic marker). The relative mean \pm s.e.m. (b) Annexin $V$ (staining intensity scored between 0 and 3) and (d) TGF- $\beta 1$ (staining intensity scored between 0 and 3 ) were then morphometrically assessed from immunohistochemically stained sections derived from each animal analyzed. Numbers in parentheses represent the

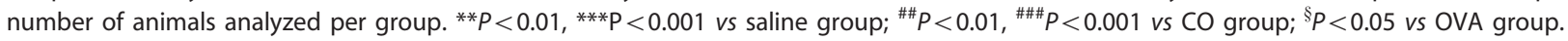

OVA-sensitized/challenged and OVA + NA-treated animals, it is likely that the cell apoptosis associated with OVAsensitized/challenged and OVA + NA-treated mice was independent of epithelial denudation and was instead associated with the corresponding levels of AWR and related fibrosis seen in these groups, respectively.

As demonstrated before, ${ }^{22}$ goblet cell metaplasia was significantly increased in OVA-sensitized/challenged mice 


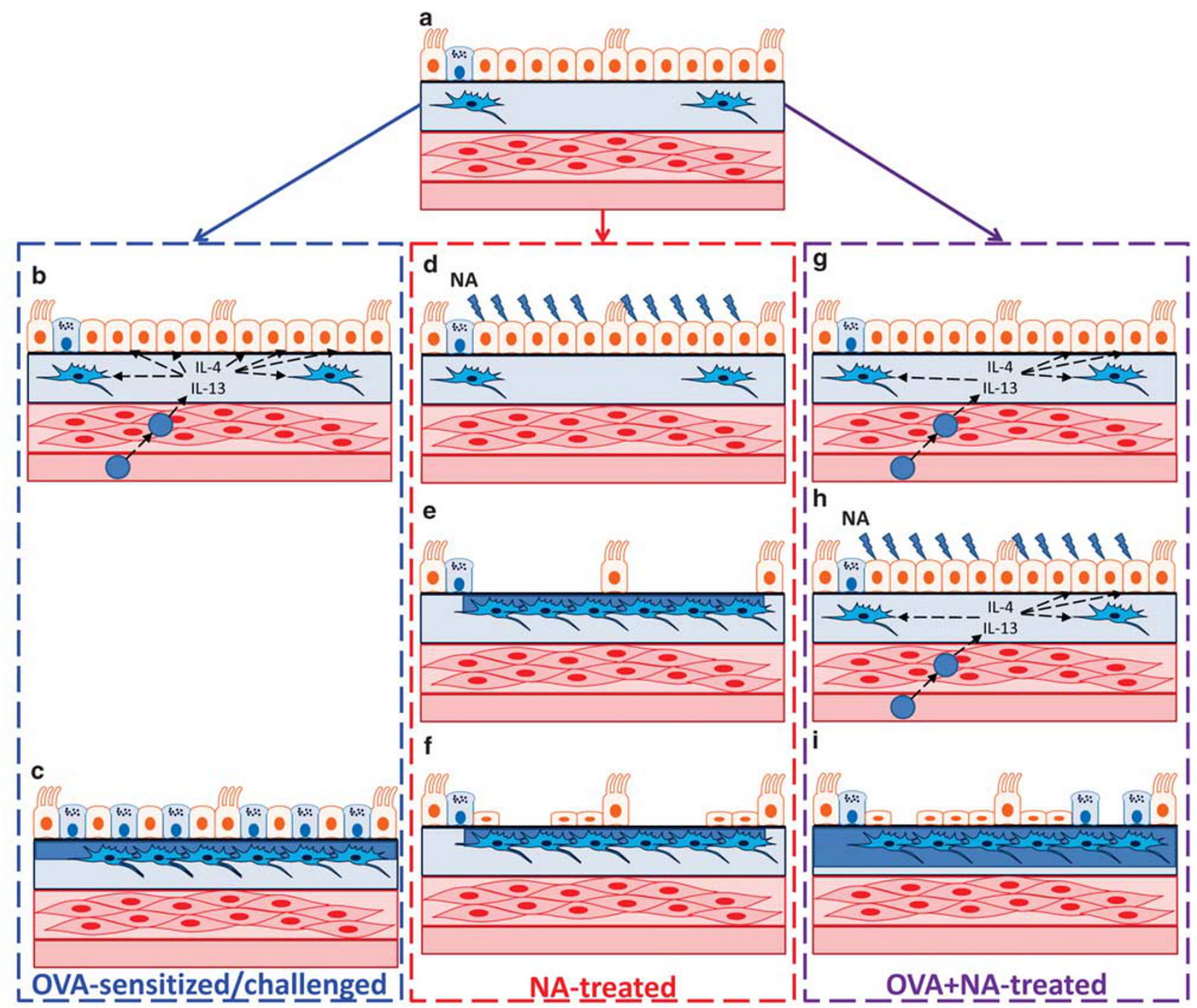

\section{Legend:}

$\longrightarrow$ Smooth muscle cell

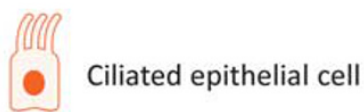

- Clara cell

G: Goblet Cell

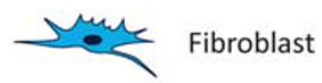

Th2 cell

Induced damage

Basement membrane
Reticular basement membrane

Collagen deposition

Subepithelial layer

Blood vessel

Figure 7 For caption please refer page 1338.

compared with saline controls. Interestingly, goblet cell metaplasia was less in OVA + NA-treated mice, compared with that measured in OVA mice. Airway epithelial thickening leads to a reduction in airway lumen size, resulting in a greater degree of AHR and corresponding AHR. ${ }^{28}$ Studies have shown that in nonfatal asthma epithelial 
Figure 7 The proposed sequence of events that occur in the OVA + NA-treated model. (a) The healthy lung contains airway epithelia that are primarily composed of Clara cells and, to a lesser extent, ciliated epithelial cells and goblet cells. (b) OVA sensitization/challenge-induced Al triggers an influx of inflammatory cells and Th2 cells that release a variety of cytokines, predominately IL-4 and IL-13 which stimulate (c) the (i) recruitment and differentiation of fibroblasts to myofibroblasts, resulting in increased collagen deposition and (ii) conversion of Clara cells into goblet cells. (d) On the other hand, administration of NA causes Clara cell death, resulting in (e) epithelial denudation. This in turn results in epithelium apoptosis/loss of cells from the epithelium, the recruitment and differentiation of fibroblasts into myofibroblasts, and increased collagen deposition. (f) By $72 \mathrm{~h}$ post NA administration, natural re-epithelialization and repair processes will be activated, resulting in the proliferation of basal cells on the exposed regions of the basement membrane. (g) In the combined model, OVA sensitization/challenge triggers the same sequences of events reported above and demonstrated in b. (h) The administration of NA on day 64 leads to limited Clara cell death (as a subset of Clara cells are already differentiated into goblet cells by OVA sensitization/challenge and epithelial denudation. (i) This in turn leads to epithelium apoptosis and exacerbated fibrosis and AHR (most likely due to OVA-induced Al and NA-induced epithelial-damage independently activating Th2 cells to secrete various factors that activate myofibroblast differentiation and myofibroblast-mediated collagen production (fibrosis). OVA-induced epithelial thickness was also maintained following NA administration.

thickness can increase by $\sim 10-100 \%,{ }^{11}$ which is consistent with the findings of this study, which demonstrated that epithelial thickness was significantly and comparably increased in OVA-sensitized/challenged and OVA + NAtreated mice by $\sim 20 \%$, relative to that in saline animals. Based on the changes in AHR measurements between the groups, it was expected that epithelial thickness in OVA + NA-treated mice would have been greater than that measured in OVA-sensitized/challenged animals. However, the levels of epithelial thickness in the combined model may have been limited to a certain extent by NA-induced re-epithelialization. The relatively large surface area of the human respiratory tract allows for more heterogeneity with areas of normal epithelium, exposed basement membrane, goblet cell metaplasia, low re-epithelialization, and thickened epithelium, as well as squamous cell metaplasia (not seen in the mouse), corresponding to varying thicknesses and stages of the damage repair cycle related to focal lesions. ${ }^{39}$

In summary, a combined OVA + NA model was established, which developed several features of human asthma to a greater extent than the chronic AAD model. The potential advantage of this combined OVA + NA-treated model (over existing models) is that it incorporates AI, epithelial damage, AWR, and AHR as part of its pathology, and therefore is more likely to undergo structural and functional changes that are more reflective of human asthma. Additionally, given the striking increase in fibrosis and AHR that was observed in the combined model, perhaps this model can now be used to evaluate novel treatments that target epithelial damage (such as epithelial repair molecules and trefoil factors); ${ }^{25}$ and related fibrosis (such as relaxin, ${ }^{40}$ pirfenidone $^{41}$ and stem cells ${ }^{42}$ ). The characterization of this model has led us to demonstrate that epithelial damage is a key contributor to airway fibrosis and related AHR, and exacerbates AIinduced AWR.

\section{ACKNOWLEDGMENTS}

This study was supported in part by a Monash University MBio Postgraduate Discovery Scholarship (MPDS) to KPP, and Monash University Mid-Career and National Health \& Medical Research Council (NHMRC) of Australia Senior Research Fellowships (APP1041766) to CSS.

\section{DISCLOSURE/CONFLICT OF INTEREST}

The authors declare no conflict of interest.

1. Tang ML, Wilson JW, Stewart AG, et al. Airway remodelling in asthma: current understanding and implications for future therapies. Pharmacol Ther 2006;112:474-488.

2. Malmstrom K, Pelkonen AS, Makela MJ. Remodeling, inflammation and airway responsiveness in early childhood asthma. Curr Opin Allergy Clin Immunol 2013;13:203-210.

3. Holgate ST. Epithelial damage and response. Clin Exp Allergy 2000;30(Suppl 1):37-41.

4. Laitinen $L A$, Heino $M$, Laitinen $A$, et al. Damage of the airway epithelium and bronchial reactivity in patients with asthma. Am Rev Respir Dis 1985;131:599-606.

5. Zhang Y, Moffatt MF, Cookson WO. Genetic and genomic approaches to asthma: new insights for the origins. Curr Opin Pulm Med 2012; 18:6-13.

6. lerodiakonou D, Postma DS, Koppelman GH, et al. E-cadherin gene polymorphisms in asthma patients using inhaled corticosteroids. Eur Respir J 2011;38:1044-1052.

7. de Boer WI, Sharma HS, Baelemans SM, et al. Altered expression of epithelial junctional proteins in atopic asthma: possible role in inflammation. Can J Physiol Pharmacol 2008;86:105-112.

8. Trautmann A, Kruger K, Akdis M, et al. Apoptosis and loss of adhesion of bronchial epithelial cells in asthma. Int Arch Allergy Immunol 2005; 138:142-150.

9. Sears MR, Taylor DR, Print CG, et al. Regular inhaled beta-agonist treatment in bronchial asthma. Lancet 1990;336:1391-1396.

10. Barnes PJ. New therapies for asthma: is there any progress? Trends Pharmacol Sci 2010;31:335-343.

11. Locke NR, Royce SG, Wainewright JS, et al. Comparison of airway remodeling in acute, subacute, and chronic models of allergic airways disease. Am J Respir Cell Mol Biol 2007;36:625-632.

12. Maes T, Provoost S, Lanckacker EA, et al. Mouse models to unravel the role of inhaled pollutants on allergic sensitization and airway inflammation. Respir Res 2010;11:7.

13. Hirota JA, Hackett TL, Inman MD, et al. Modeling asthma in mice: what have we learned about the airway epithelium? Am J Respir Cell Mol Biol 2011;44:431-438.

14. Van Winkle LS, Buckpitt AR, Nishio SJ, et al. Cellular response in naphthalene-induced Clara cell injury and bronchiolar epithelial repair in mice. Am J Physiol 1995;269:L800-L818.

15. Elizur A, Adair-Kirk TL, Kelley DG, et al. Clara cells impact the pulmonary innate immune response to LPS. Am J Physiol Lung Cell Mol Physiol 2007;293:L383-L392.

16. Gail DB, Lenfant CJ. Cells of the lung: biology and clinical implications. Am Rev Respir Dis 1983;127:366-387.

17. Singh G, Katyal SL. Clara cells and Clara cell $10 \mathrm{kD}$ protein (CC10). Am J Respir Cell Mol Biol 1997;17:141-143.

18. Melgert BN, Postma DS, Kuipers I, et al. Female mice are more susceptible to the development of allergic airway inflammation than male mice. Clin Exp Allergy 2005;35:1496-1503. 
19. Corteling R, Trifilieff A. Gender comparison in a murine model of allergen-driven airway inflammation and the response to budesonide treatment. BMC Pharmacol 2004;4:4.

20. Kumar RK, Herbert C, Foster PS. The 'classical' ovalbumin challenge model of asthma in mice. Curr Drug Targets 2008;9: 485-494.

21. Zosky GR, Sly PD. Animal models of asthma. Clin Exp Allergy 2007; 37:973-988.

22. Temelkovski J, Hogan SP, Shepherd DP, et al. An improved murine model of asthma: selective airway inflammation, epithelial lesions and increased methacholine responsiveness following chronic exposure to aerosolised allergen. Thorax 1998;53:849-856.

23. Royce SG, Li X, Tortorella S, et al. Mechanistic insights into the contribution of epithelial damage to airway remodeling: novel therapeutic targets for asthma. Am J Respir Cell Mol Biol 2014;50: 180-192.

24. Gallop PM, Paz MA. Posttranslational protein modifications, with special attention to collagen and elastin. Physiol Rev 1975;55: 418-487.

25. Royce SG, Lim C, Muljadi RC, et al. Trefoil factor-2 reverses airway remodeling changes in allergic airways disease. Am J Respir Cell Mol Biol 2013:48:135-144.

26. Phipps $S$, Benyahia $F, O u ~ T T$, et al. Acute allergen-induced airway remodeling in atopic asthma. Am J Respir Cell Mol Biol 2004;31: 626-632.

27. Holgate ST, Lackie PM, Davies DE, et al. The bronchial epithelium as a key regulator of airway inflammation and remodelling in asthma. Clin Exp Allergy 1999;29(Suppl 2):90-95.

28. Elias JA, Zhu Z, Chupp G, et al. Airway remodeling in asthma. J Clin Invest 1999;104:1001-1006.

29. Tatler AL, Jenkins G. TGF-beta activation and lung fibrosis. Proc Am Thorac Soc 2012;9:130-136.

30. Boulet LP, Laviolette $M$, Turcotte $H$, et al. Bronchial subepithelial fibrosis correlates with airway responsiveness to methacholine. Chest 1997;112:45-52.
31. Chetta A, Foresi A, Del Donno $M$, et al. Airways remodeling is a distinctive feature of asthma and is related to severity of disease. Chest 1997:111:852-857.

32. Hoshino M, Nakamura Y, Sim JJ, et al. Inhaled corticosteroid reduced lamina reticularis of the basement membrane by modulation of insulin-like growth factor (IGF)-I expression in bronchial asthma. Clin Exp Allergy 1998;28:568-577.

33. Moreno RH, Hogg JC, Pare PD. Mechanics of airway narrowing. Am Rev Respir Dis 1986;133:1171-1180.

34. Wiggs BR, Bosken C, Pare PD, et al. A model of airway narrowing in asthma and in chronic obstructive pulmonary disease. Am Rev Respir Dis 1992;145:1251-1258.

35. Karagiannis TC, Li X, Tang MM, et al. Molecular model of naphthaleneinduced DNA damage in the murine lung. Hum Exp Toxicol 2012; 31:42-50.

36. Atherton $\mathrm{HC}$, Jones $\mathrm{G}$, Danahay $\mathrm{H}$. IL-13-induced changes in the goblet cell density of human bronchial epithelial cell cultures: MAP kinase and phosphatidylinositol 3-kinase regulation. Am J Physiol Lung Cell Mol Physiol 2003;285:L730-L739.

37. Dabbagh $\mathrm{K}$, Takeyama $\mathrm{K}$, Lee $\mathrm{HM}$, et al. IL-4 induces mucin gene expression and goblet cell metaplasia in vitro and in vivo. J Immunol 1999;162:6233-6237.

38. Erjefalt JS, Korsgren M, Nilsson MC, et al. Prompt epithelial damage and restitution processes in allergen challenged guinea-pig trachea in vivo. Clin Exp Allergy 1997;27:1458-1470.

39. Crosby LM, Waters CM. Epithelial repair mechanisms in the lung. Am J Physiol Lung Cell Mol Physiol 2010;298:L715-L731.

40. Royce SG, Miao YR, Lee M, et al. Relaxin reverses airway remodeling and airway dysfunction in allergic airways disease. Endocrinology 2009;150:2692-2699.

41. Gomer RH. New approaches to modulating idiopathic pulmonary fibrosis. Curr Allergy Asthma Rep 2013;13:607-612.

42. Moodley $\mathrm{Y}$, Atienza D, Manuelpillai $\mathrm{U}$, et al. Human umbilical cord mesenchymal stem cells reduce fibrosis of bleomycin-induced lung injury. Am J Pathol 2009;175:303-313. 